\title{
Electromagnetic Interference Reflectivity of Nanostructured Manganese Ferrite Reinforced Polypyrrole Composites
}

\author{
Himel Chakraborty ${ }^{\dagger}$ \\ School of Materials Science and Engineering, Bengal Engineering and Science University, Shibpur, Howrah, West Bengal \\ 711103, India \\ Sumit Chabri and Nandagopal Bhowmik \\ Department of Metallurgy and Materials Engineering, Bengal Engineering and Science University, Shibpur, Howrah, West \\ Bengal 711103, India
}

Received April 22, 2013; Revised August 3, 2013; Accepted October 24, 2013

\begin{abstract}
Nano-size manganese ferrite reinforced conductive polypyrrole composites reveal a core-shell structure by in situ polymerization, in the presence of dodecyl benzene sulfonic acid as the surfactant and dopant, and iron chloride as the oxidant. The structure and magnetic properties of manganese ferrite nano-fillers were measured, by using X-ray diffraction and vibrating sample magnetometer. The morphology, microstructure, and conductivity of the composite were characterized by transmission electron microscopy, Fourier transform infrared spectroscopy, and four-wire technique. The microwave-absorbing properties of composites reinforcement dispersed in resin coating with the coating thickness of $1.2 \mathrm{~nm}$ were investigated, by using vector network analyzers, in the frequency range of $8 \sim 12 \mathrm{GHz}$. A reflection loss of $-8 \mathrm{~dB}$ was observed at $10.5 \mathrm{GHz}$.
\end{abstract}

Keywords: Nanocomposite, Magnetic materials, EMI shielding

\section{INTRODUCTION}

Polymer composites with both electrical and ferromagnetic properties have attracted significant consideration. The conducting polymer composites are attractive, due to their multifunctional applications in batteries, electro-chemical display devices, electric magnetic shielding material, microwave-absorbing materials, etc. [1-3]. Polypyrrole (PPy) is one of the conducting polymers, because of its high conductivity, electrical properties, and ease of production $[4,5]$. It is well known that PPy matrix can effectively reflect electro-magnetic waves generated from an electric source, and the electro-magnetic waves from a magnetic

${ }^{\dagger}$ Author to whom all correspondence should be addressed: E-mail: himelchakraborty@matsc.becs.ac.in

Copyright @2013 KIEEME. All rights reserved. This is an open-access article distributed under the terms of the Creative Commons Attribution Non-Commercial
License (http://creativecomms.org/licenses/by-nc/3.0) which permits unrestricted noncommercial use, License (http://creativecommons.org/licenses/by-nc/3.0) which permits unrestricted
distribution, and reproduction in any medium, provided the original work is properly cited. source can be shielded by using magnetic materials [6,7]. Thus, the reinforcement of magnetic fillers into PPy matrix can generate multifunctional composites, with new physical properties [8]. The research on microwave-absorbing materials continues to attract much attention, and ferrites is a popular material. But the use of ferrite-based absorbers requires higher performance at higher frequencies, such as X-band $[9,10]$. Among the conventional microwave absorption materials, the spinel-type ferrites are popular. However, spinel-type ferrites show Snoek's limit, and the magnetic loss decreases at giga-hertz range [11-14]. Earlier, spinel ferrites have been most frequently utilized as absorbing materials, in various forms. Manganese ferrite $\left(\mathrm{MnFe}_{2} \mathrm{O}_{4}\right)$ is a common spinel ferrite material, and has been widely used in microwave and magnetic recording applications. However, it has been shown that magnetic composites are useful as microwaveabsorbing materials, due to their advantages in respect of lightweight, low cost, design flexibility, and microwave properties. In the present work, $\mathrm{MnFe}_{2} \mathrm{O}_{4}$ is reinforced in the PPy matrix, and 
its effect on electrical and ferromagnetic properties is compared with the pristine form.

\section{EXPERIMENTS}

The chemicals, including metal salts, hexamethylenetetramine (HMTA), potassium persulfate (KPS) and ethylene glycol (EG), were analytical grade from Sigma, and were used without further purification. Water was deionized, doubly distilled, and deoxygenated, prior to use. The styrene and methacrylic acid were purchased analytical grade from Sigma, and were distilled to remove the inhibitor. The pyrrole monomer was distilled twice, under reduced pressure. The DBSA and acrylic resin were of industria grade. The other reagent, including iron chloride $\left(\mathrm{FeCl}_{3}\right)$, was of analytical grade Sigma.

Negatively charged PS spheres with average diameter 230 $\mathrm{nm}$, which were used as core particles, were prepared by a freeemulsion polymerization method. In a typical experiment, 10 $\mathrm{ml}$ styrene, $2 \mathrm{ml}$ methacrylic acid, and $0.055 \mathrm{~g}$ KPS were added to a flask with $100 \mathrm{ml}$ de-ionized water. To eliminate oxygen effects, the solution was purged with nitrogen, before the process was initiated. The mixture was heated to $75^{\circ} \mathrm{C}$, and stirred with a magnetic stirrer. The polymerization was continued for $24 \mathrm{~h}$, and in the whole procedure, the nitrogen was purged. The concentration of PS spheres in solution was $80 \mathrm{mg} / \mathrm{ml}$, which was calculated by drying $4 \mathrm{ml}$ colloid solution, and weighing the remained solids.

The coating technique consisted of controlled hydrolysis of aqueous solutions of ferrous chloride and other divalent metal salts, in the presence of polystyrene latexes. In a typical preparation process, $2 \mathrm{ml}$ PS colloid solution was diluted with $300 \mathrm{ml}$ deoxygenated distilled water, and then mixed with the metal salts solution, which contained $10 \mathrm{~m} . \mathrm{mol} \mathrm{FeCl}$ and $5 \mathrm{~m} . \mathrm{mol} \mathrm{MnCl}_{2}$. After dispersal under ultra-sonic for several minutes, the mixture was incorporated with $4 \mathrm{~g}$ HMTA and $0.5 \mathrm{~g}$ potassium nitrate, and heated to $90^{\circ} \mathrm{C}$, under gentle stirring. After $3 \mathrm{~h}$, the system was cooled to room temperature. The solution was poured into excess distilled water, then magnetic particles were deposited, using a magnetic field. The precipitate was washed with distilled water several times, and then dried in oven, at $75^{\circ} \mathrm{C}$ for $24 \mathrm{~h}$. In addition, to modify the surface chemical properties of the composites magnetic spheres, $5 \mathrm{ml}$ ethylene glycol (EG) was added into the reaction solution, before the incorporation of HMTA.

The $\mathrm{MnFe}_{2} \mathrm{O}_{4}$-PPy core-shell composites were prepared by in situ polymerization, in the presence of DBSA as the surfactant and dopant, and $\mathrm{FeCl}_{3}$ as the oxidant. The DBSA was dissolved in distilled water with vigorous stirring for about $30 \mathrm{~min}$, and then $\mathrm{MnFe}_{2} \mathrm{O}_{4}$ nano-fillers (0.73 g) were added to the DBSA solution, under stirring for approximately $60 \mathrm{~min}$. Then $5 \mathrm{ml}$ of pyrrole monomer was added to the suspension, and stirred for $30 \mathrm{~min}$. $\mathrm{MnFe}_{2} \mathrm{O}_{4}$ nano-fillers were dispersed well in the mixture of pyrrole/DBSA under ultra-sonication for $120 \mathrm{~min}$, and $24 \mathrm{~g}$ of $\mathrm{FeCl}_{3}$ in $75 \mathrm{ml}$ deionized water was slowly added drop wise to the mixture, with constant stirring. The polymerization with stirring under an ice-water bath was allowed to proceed for $4 \mathrm{~h}$. The composite was obtained by filtering and washing the suspension with de-ionized water and ethanol, respectively. The obtained black powder with the content of 10 wt.\% $\mathrm{MnFe}_{2} \mathrm{O}_{4}$ was dried under vacuum chamber for $24 \mathrm{~h}$.

The morphology of coated particles and composite was observed by transmission electron microscopy (TEM), with a Hitachi instrument operated at an accelerating voltage of $10 \mathrm{kV}$. The $\mathrm{X}$-ray powder diffraction (XRD) patterns of the nanoparticles assemblies were collected on a Philips-PW 1800 with $\mathrm{Cu}-\mathrm{K}$ radiation, under CuK $\alpha$ radiation $(\lambda=1.5406 \AA)$. The fourier transform infrared spectroscopy (FTIR) spectra were recorded on a PerkinElmer spectrum FTIR, using KBr pellets. The M-H hysteresis loops were measured by vibrating sample magnetometer (VSM) (Riken Denshi Co. Ltd., Japan). Electromagnetic radiation reflectivity measurements, over the frequency range of $8 \sim 12 \mathrm{GHz}$, were done using a waveguide, coupled to an Agilent Synthesized Sweeper model 8,375 A, and a HP 7000 spectrum analyzer.

\section{RESULTS AND DISCUSSION}

A TEM micrograph of the $10 \mathrm{wt} \% \mathrm{MnFe}_{2} \mathrm{O}_{4}$ reinforced PPy composite is shown in Fig. 1. The microstructure of the composite shows the complex networking of $\mathrm{MnFe}_{2} \mathrm{O}_{4}$ fillers in the PPy matrix. The inset of Fig. 1 shows EDS composition of the $\mathrm{MnFe}_{2} \mathrm{O}_{4}$ particles, indicating the high-quality dispersion of the fillers within the PPy matrix.

Figure 2 shows the XRD spectrum of manganese ferrites nanofillers. It can be clearly observed from the XRD spectrum that the ferrite shells are phase-pure spinel structure in all cases, according to the standard XRD spectra of spinel ferrite $\mathrm{Fe}_{3} \mathrm{O}_{4}(2 \theta=35.5$, $43.7,57.0,62.4,74.0,78.0)$, Mn ferrite $(2 \theta=33.0,71.0,86.4)$, and $\mathrm{NaCl}(2 \theta=75.5)$. The average crystallite size was calculated using JCPDS, through the diffraction peaks from Scherer's equation [15].

In Fig. 3 show the FT-IR spectra of $\mathrm{MnFe}_{2} \mathrm{O}_{4}$ and $\mathrm{PPy}-\mathrm{MnFe}_{2} \mathrm{O}_{4}$ composite. It was observed from Fig. 3 that the peak at $571 \mathrm{~cm}^{-1}$ is the intrinsic vibrations of manganese ferrite. The characteristic peaks of styrene occur at $811,865,1,009$ to $1,382,1,458$ and $1,638 \mathrm{~cm}^{-1}$. The peaks at 811 and $865 \mathrm{~cm}^{-1}$ are related to the $\mathrm{C}-\mathrm{H}$ outer bending vibrations. The peak at $1458 \mathrm{~cm}^{-1}$ is attributed to the characteristic $\mathrm{C}=\mathrm{C}$ stretching ring. The peak at $1,638 \mathrm{~cm}^{-1}$ is attributed to the styrene ring.

As shown in (b) of Fig 3, the characteristic peaks of PPymanganese ferrite composite occur at 576, 670, 908, 959, 1,006, $1,038,1,188,1,313,1,461,1,555$ and $2,920 \mathrm{~cm}^{-1}$. However, the characteristic peaks of $\mathrm{MnFe}_{2} \mathrm{O}_{4}$ for F-O and Mn-O stretching vibrations can be observed at wave numbers 576 and $670 \mathrm{~cm}^{-1}$. The peaks at 908 to $1,006 \mathrm{~cm}^{-1}$ are attributed to the p-disubstituted aromatic ring $\mathrm{C}-\mathrm{H}$ out-of-plane bending. The peak around 1,038 $\mathrm{cm}^{-1}$ is associated with vibrational modes of $\mathrm{N}=\mathrm{Q}=\mathrm{N}$ ( $\mathrm{Q}$ refers to the quinonic type rings), indicating that PPy is formed in our sample. The peaks at 1,461 and $1,555 \mathrm{~cm}^{-1}$ are attributed to the characteristic $\mathrm{C}=\mathrm{C}$ and $\mathrm{C}-\mathrm{N}$ stretching of polypyrrole ring; the peaks at 1,188 and $1,313 \mathrm{~cm}^{-1}$ correspond to $\mathrm{N}-\mathrm{H}$ bending and asymmetric C-N stretching modes of the PPy ring.

Figures 4 (a) (b) show the magnetization (M) versus the applied magnetic field (H) for $\mathrm{MnFe}_{2} \mathrm{O}_{4}$ and $\mathrm{MnFe}_{2} \mathrm{O}_{4} / \mathrm{PPy}$ composite $(10 \mathrm{wt} \%)$, respectively. The magnetic properties of the ferrite-coated PS latex were analyzed by room temperature VSM, with an applied field of $-10 \leq H \leq 10 \mathrm{kOe}$. It can be inferred from the hysteresis loops that all the composite magnetic spheres are magnetically soft at room temperature. The value of saturation magnetization $(\mathrm{Ms})$ is about $68 \mathrm{emu} / \mathrm{g}$, and the remnant magnetization $(\mathrm{Mr}$ ) and coercivity field are $18 \mathrm{emu} / \mathrm{g}$ and $110 \mathrm{Oe}$, respectively. Figure 4 (b) shows clear saturation $-10 \leq H \leq 10 \mathrm{kOe}$, with saturation magnetization $(M s)$ of about $10 \mathrm{emu} / \mathrm{g}$, and remnant magnetization $(\mathrm{Mr})$ of about $3.4 \mathrm{emu} / \mathrm{g}$, for nanocomposite that is lower than pure manganese ferrite nano-fillers, so the magnetization curve of the sample shows weak ferromagnetic behavior, with slender hysteresis. The magnetic properties of composites containing magnetite or ferrite nano-fillers have been believed to be highly dependent on the sample shape, crystallinity, and value of magnetic nano-fillers, so that they can be adjusted, to obtain optimum properties. The magnetic properties of composite and ferrite nano-fillers showed soft magnetization behavior. 


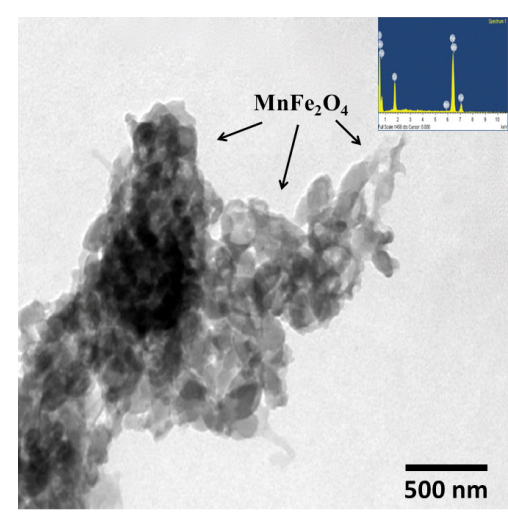

Fig. 1. A TEM micrograph of $10 \mathrm{wt} \% \mathrm{MnFe}_{2} \mathrm{O}_{4} / \mathrm{PPy}$ composite.

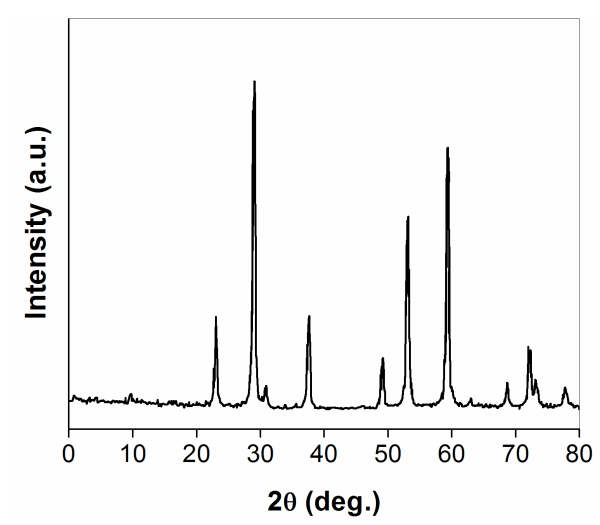

Fig. 2. X-ray diffraction of manganese ferrites nano-fillers.

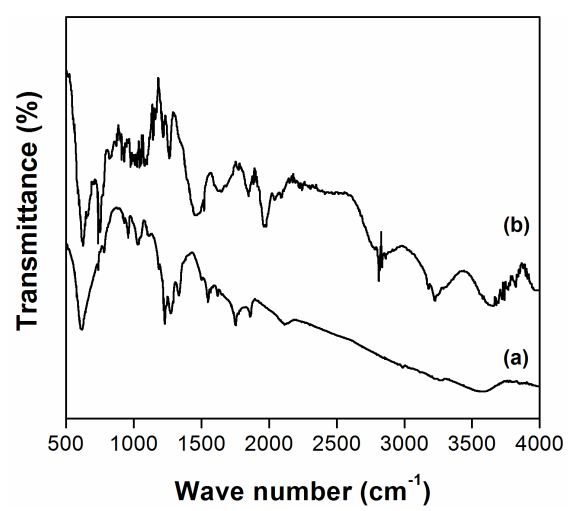

Fig. 3. FT-IR spectra of (a) $\mathrm{MnFe}_{2} \mathrm{O}_{4}$ nano-fillers and (b) $\mathrm{MnFe}_{2} \mathrm{O}_{4} / \mathrm{PPy}$ composite.

In Fig. 5, the results of reflectivity (in decibels, $d B$ ) are presented as a function of frequency (GHz). In the present study, the permittivity data are not available for the samples; thus, comparison with theoretical reflection curves is not possible. High radiation attenuation values (ca. $-9.5 \mathrm{~dB}$ ), in the frequency range of 11 12 GHz, were obtained in the PPy matrix. Also, the composite containing $10 \mathrm{wt} \%$ of composite presented radiation attenuation values between -1 and $-3 \mathrm{~dB}$, in the frequency range of $8 \sim 12 \mathrm{GHz}$. In this case, the composite exhibits a broadband behavior, with microwave radiation absorption of $80 \%$. We can observe that the incorporation of $\mathrm{MnFe}_{2} \mathrm{O}_{4}$ as reinforcement in the PPy matrix leads to a shift of the attenuation values to lower frequencies, and the composite attained high radiation attenuation values
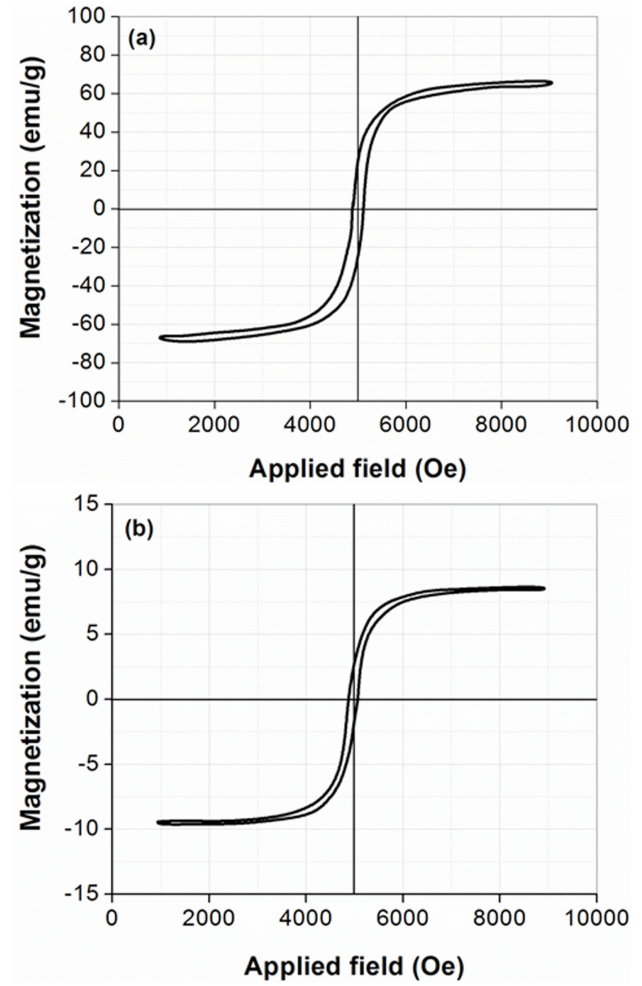

Fig. 4. The magnetic hysteresis loop of (a) $\mathrm{MnFe}_{2} \mathrm{O}_{4}$ and (b) $\mathrm{MnFe}_{2} \mathrm{O}_{4}$ / PPy composite.

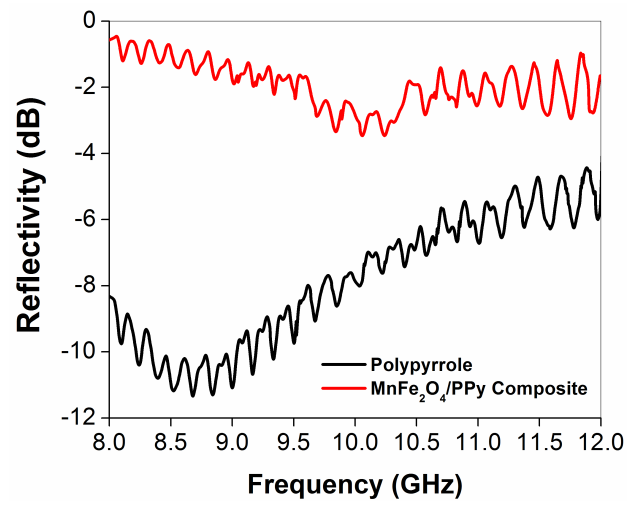

Fig. 5. Reflectivity of the polypyrrole and $\mathrm{MnFe}_{2} \mathrm{O}_{4} / \mathrm{PPy}$ composite.

(ca.-10 dB), in the frequency range of $8 \sim 10 \mathrm{GHz}$, corresponding to an attenuation between 75 and $95 \%$ of the incident radiation. This is due to the dielectric characteristics of polypyrrole. In other words, minimal reflection of the microwave power or matching conditions occurs. This shows that by reinforcement of $\mathrm{MnFe}_{2} \mathrm{O}_{4}$ nano-fillers, the attenuation peak frequency of composites can be manipulated.

\section{CONCLUSIONS}

The $\mathrm{MnFe}_{2} \mathrm{O}_{4}$ magnetic nano-fillers are of size $25.43 \mathrm{~nm}$. PPymanganese ferrite composite with magnetic behavior is successfully synthesized by in situ polymerization of pyrrole, in the presence of $\mathrm{MnFe}_{2} \mathrm{O}_{4}$ nano-fillers. The results of spectro-analysis indicate that possible interaction occurred between the PPy chains and $\mathrm{MnFe}_{2} \mathrm{O}_{4}$ particles. A minimum reflectivity of $-0.89 \mathrm{~dB}$ 
was observed at $11.7 \mathrm{GHz}$, with thickness of $1.2 \mathrm{~nm}$ of composite. The absorption peak frequency can be easily manipulated, by changing the thickness of the microwave absorber. Also, the ease of processing of the composites permits making multi-layers with good reflectivity performance.

\section{ACKNOWLEDGMENTS}

Author Himel Chakraborty would like to thank the Human Resource Development Group, of the Council for Scientific and Industrial Research, India, (File no. (003)0061/EMR-I/2009), for providing financial support.

\section{REFERENCES}

[1] J. Y. Shin, H. D. Park, K. J. Choi, K. W. Lee, J. Y. Lee, J. W. Hong. Trans. Electr. Electron. Mater. 10, 97 (2009) [DOI: http://dx.doi. org/1229-7607/2009/10(3)/97/5].

[2] K. T. Mathew, A. V. P. Kumar, H. John. International Symposium on Electromagnetic Compatibility, 2006. EMC 06. Annual Report Conference. 2, 443 (2006) [ DOI: http://dx.doi.org/10.1109/ ISEMC.2006.1706344]

[3] E. M. I. M. Ekanayake, D. M. G Preethichandra, K. Kaneto. IEEE Trans. on Instrument. and Measurement. 57, 1621 (2008) [DOI: http://dx.doi.org/10.1109/TIM.2008.922068].

[4] E. M. I. M. Ekanayake, D. M. G Preethichandra, K. Kaneto. Conf. Proceed. Instrument. and Measurement Technol. 2007. IMTC 07. Annual Report Conference. 1 (2007) [DOI: http://dx.doi org/10.1109/IMTC.2007. 378998].
[5] J. J. Park. Trans. Electr. Electron. Mater. 14, 43 (2013) [DOI: http://dx.doi.org/10.4313/TEEM.2013.14.1.43].

[6] E. M. I. M. Ekanayake, D. M. G Preethichandra, K. Kaneto. Industrial and Information Systems. ICIIS 07. 63 (2007) [DOI: http://dx.doi.org/10.1109/ICIINFS.2007.4579149].

[7] S. C. Byeon, H. J. Je, K. S. Hong. Trans. on Magnetics 36, 371 (2000) [DOI: http://dx.doi.org/10.1109/20.822549].

[8] A, Ghasemi, V. Sepelak, X. Liu, A. Morisako. Trans. on Magnetics 45, 2456 (2009) [DOI: http://dx.doi.org/10.1109/ TMAG.2009.2018611].

[9] M. Damnjanovic, L. Zivanov, G. Stojanovic. EUROCON 07. 905 (2007) [DOI: http://dx.doi.org/10.1109/EURCON.2007.4400453].

[10] P. M. Raj, H. Sharma, D. Mishra, K. P. Murali, K. Han, M. Swaminathan, R. R. Tummala. Nanotechnol. Mag. 6, 18 (2012) [DOI: http://dx.doi.org/10.1109/MNANO.2012.2203878].

[11] Molenberg, J. Thomassin, E. Ferain, C. Detrembleur, I. Huynen. Eur. Microwave Conf. EuMC 09. Annual Report Conference. 1045 (2009) [DOI: http://dx.doi.org/10.1109/ EUMC.2009.1107635].

[12] R. L. Moore. Inter. Microwave Sympos. Digest. IEEE MTT-S 11. Annual Report Conference. 1 (2011) [DOI: http://dx.doi. org/10.1109/MWSYM.2011.5972563].

[13] V. G. Harris. Trans. on Magnetics 48, 1075 (2012) [DOI: http:// dx.doi.org/10.1109/TMAG.2011.2180732].

[14] P. Bollen, N. Quievy, C. Detrembleur, J. M. Thomassin, C. Bailly, T. Pardoen, I. Huynen. Inter. Sympos. On Electromagnetic Compatibility, EMC EUROPE 12. Annual Report Conference. 1 (2012) [DOI: http://dx.doi.org/10.1109/EMCEurope.2012.6396808].

[15] M. H. Fathi, A. Hanifi. Mater. Lett. 61, 18 (2007) [DOI: http:// dx.doi.org/10.1016/j.matlet.2007.01.028]. 